\title{
Functional extreme rainfall data in Petaling Jaya
}

\begin{abstract}
This research implements two important main fields of the study which is functional data analysis and extreme value theory. The aims of this study are to convert the extreme rainfall data into functions and to propose method development for functional data analysis in extreme value theory. Functional data analysis is one of the techniques to convert or transform discrete observations into functions or curves. The daily rainfall data in Petaling Jaya from 1974 to 2003 will be tested on extreme value theory approach which is block maxima in order to practice the functional data analysis technique. The least squares and akaike criteria information method are used to smooth the fourier basis of the functional data analysis. A descriptive statistics which represents the behavior of a rainfall data for Petaling Jaya is analyzed and illustrated by a smooth curve from functional data analysis. This study indicates that there is an increase in rates of the rainfall in Petaling Jaya for the recent period compared to the last 30 years data both in the mean and standard deviation values.
\end{abstract}

Keyword: Functional data analysis; Extreme value theory; Extreme rainfall data 